\title{
Detection of anti-ADAM 10 antibody in serum of a patient with pulmonary fibrosis associated with dermatomyositis
}

\author{
Jiro Fujita, Tamotsu Takeuchi, Naomi Dobashi, Yuji Ohtsuki, Michiaki Tokuda, \\ Jiro Takahara
}

First Department of Internal Medicine, Kagawa Medical University, Kagawa, Japan

J Fujita

N Dobash

M Tokuda

J Takahara

Second Department of Pathology, Kochi

Medical School, Kochi,

Japan

T Takeuchi

Y Ohtsuki

Correspondence to: Dr J Fujita, First

Department of Internal

Medicine, Kagawa Medical

University, 1750-1,

Miki-cho, Kita-gun, Kagawa,

761-0793, Japan.

Accepted for publication 21 July 1999

\begin{abstract}
Objectives-It has been suggested that the humoral immune system plays a part in the pathogenesis of pulmonary fibrosis. Although circulating autoantibodies to lung protein(s) have been suggested, few lung proteins have been characterised. The purpose of this study is to determine the antigen recognised by serum of a patient with pulmonary fibrosis associated with dermatomyositis.
\end{abstract}

Methods-To accomplish this, anti-small airway epithelial cell (SAEC) antibody in a patient's serum was evaluated using a western immunoblot.

Results-An autoantibody against SAEC was found, and the antigen had a molecular weight of $62 \mathrm{kDa}$. Using the patient's serum, clones from the normal lung cDNA library were screened and demonstrated that anti-SAEC antibody in the patient's serum was against ADAM (A disintegrin and metalloprotease) 10 .

Conclusion-This is the first report that demonstrates the existence of anti-ADAM 10 antibody in a patient with pulmonary fibrosis associated with dermatomyositis. (Ann Rheum Dis 1999;58:770-772)

Idiopathic pulmonary fibrosis (IPF) and pulmonary fibrosis associated with a collagen vascular disorder are inflammatory lung diseases of unknown aetiology that are characterised by the accumulation of neutrophils and mononuclear cells. ${ }^{1}$ The presence of autoantibodies in patients with IPF has been previously predicted and investigated. ${ }^{2-9}$ The nature and location of the antigen(s) are believed to be associated with pulmonary epithelial lining cells and, perhaps, with capillary endothelial cells on the basis of ultrastructural studies ${ }^{2}$ and a previous demonstration of immune complexes at these sites. ${ }^{3}$ It has been reported that the sera from patients with IPF are positive on cryostat sections of lung tissues by indirect immunofluorescence techniques. ${ }^{4}$ Other investigators using ELISA techniques have found antibodies to the nuclear antigen topoisomerase II $(170 \mathrm{kDa})$ in $38 \%$ of patients ${ }^{5}$ and hepa- titis $\mathrm{C}$ virus in $28 \%$ of patients. ${ }^{6}$ Wallace et al have reported that circulating IgG autoantibodies to $70-90 \mathrm{kDa}$ protein(s) that are associated with alveolar epithelial lining cells are detected in sera from patients with CFA. ${ }^{78}$ Recently, we demonstrated anti-cytokeratin 8 antibody in sera of patients with pulmonary fibrosis. ${ }^{9}$ These findings suggest that there are several pulmonary autoantigens to which autoantibodies in patient's sera reacted.

We examined a patient with pulmonary fibrosis associated with dermatomyositis. As we hypothesised that anti-epithelial cell antibodies may play a part in the pathogenesis of pulmonary fibrosis, ${ }^{9}$ we evaluated the existence of anti-small airway epithelial cell (SAEC) antibodies by a western immunoblot. In addition, serum from this patient was used to isolate cDNA clones encoding autoantigens. Autoantibodies were found to target ADAM (A disintegrin and metalloprotease) $10 .{ }^{10-14}$ ADAM 10 was first identified in this study as an autoantigen in the patient with pulmonary fibrosis associated with dermatomyositis.

\section{Methods}

PATIENT AND SERA

A Japanese woman (YF) had been healthy until she was referred to Kagawa Medical University for evaluation of muscle weakness and cough at the age of 63 (in 1992). On physical examination, she was afebrile with a respiratory rate of $18 / \mathrm{min}$, blood pressure of $90 / 60 \mathrm{~mm} \mathrm{Hg}$, and a regular pulse rate of $86 / \mathrm{min}$. The chest was symmetric, and bibasilar, coarse crackles were ausculated. Arterial blood gas analysis showed $\mathrm{PO}_{2}$ of $70 \mathrm{~mm} \mathrm{Hg}, \mathrm{PCO}_{2}$ of $41 \mathrm{~mm} \mathrm{Hg}$, and $\mathrm{pH}$ 7.43. Lung function studies showed \%vital capacity $44.1 \%$ and FEV1\% 91.7\%. High resolution computed tomography showed interstitial and patchy parenchymal opacification in both lungs, predominantly in the middle and lower lung zones. Scattered ground glass opacities were also observed. No apparent honeycomb formation was observed. Although open lung biopsy was not performed, she was diagnosed to have interstitial pneumonia (possibly non-specific interstitial pneumonia) associated with dermatomyositis. She was diagnosed with pulmonary fibrosis associated with 


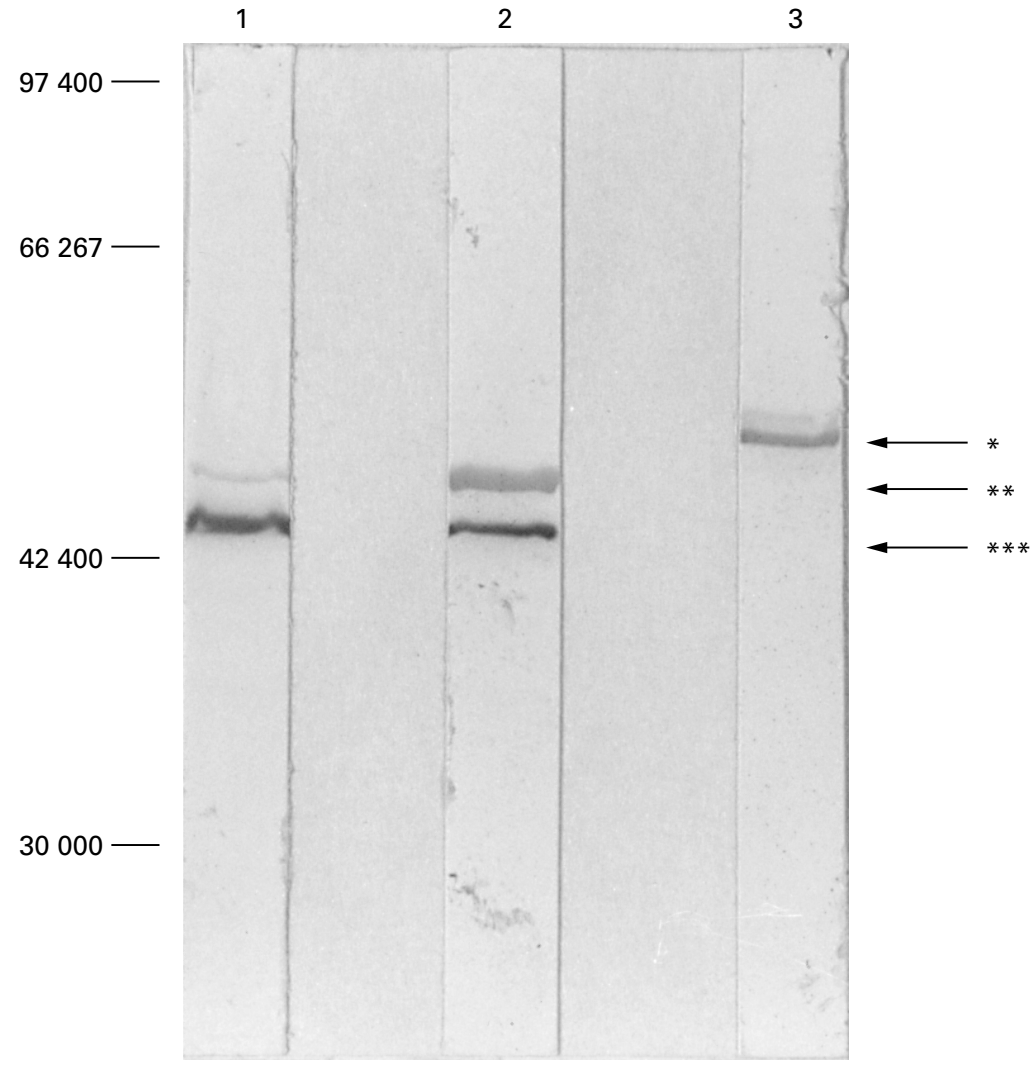

Figure 1 Western immunoblot analysis using the patient's sera against lysates of PC9, A549, and SAEC cell lines. Lane 1; PC9, lane 2; A549; lane 3; SAEC cell line. In SAEC cell line, only one band (arrow *) that has a molecular weight of $62 \mathrm{kDa}$, which is the same as ADAM 10, is demonstrated. In PC9 and A549 cell lines, two bands (arrows ** and $\star \star \star)$ are demonstrated. One antigen, which has a molecular weight of $54 \mathrm{kDa}$ (arrow ${ }^{\star *}$ ), was previously identified as cytokeratin 8 . Arrow ${ }^{\star * \star}$ is not characterised.

dermatomyositis after clinical studies including muscle biopsy. Oral dexamathasone was given to reduce her symptoms. Anti-DNA, RNP, Sm, SS-A, SS-B, Scl-70, Jo-1 antibodies were not found. No clinical symptoms or laboratory data associated with systemic autoimmune disease other than positive ANA were seen.

CELL LINES

We used cell lines as follows; A549, PC9, and SAEC cell lines. A549 and PC9 (derived from adenocarcinoma of the lung) were cultured in RPMI-1640 with $10 \%$ fetal calf serum. SAEC cell line (derived from normal small airway epithelial cells) was purchased from Sanko Junyaku Co, Ltd, and cultured in serum free medium (CCMD160, CC-3119 SABM, Sanko Junyaku Co, Ltd) with several growth factors (CC-4124, Sanko Junyaku Co, Ltd) according to the manufacturer's instruction.

\section{SDS-PAGE ELECTROPHORESIS AND WESTERN} BLOTTING

Lysates of cell lines were mixed with sodium dodecyl sulphate (SDS 2.0\%) and heated $\left(100^{\circ} \mathrm{C}, 5 \mathrm{~min}\right)$. The samples were then applied to a SDS polyacrylamide gel (gradient is lineal from $10 \%$ to $20 \%$ ), electrophoresed $(60 \mathrm{~mA}$, $120 \mathrm{~min}$ ), fixed in $50 \%$ methanol, $10 \%$ acetic acid, and stained with Coomassie Blue. Standard molecular weight markers purchased from Daiichi-Kagaku (Tokyo, Japan) were comprised of egg lysozyme (MW 14 400), trypsin inhibitor (MW 20 100), carbonic anhydrase

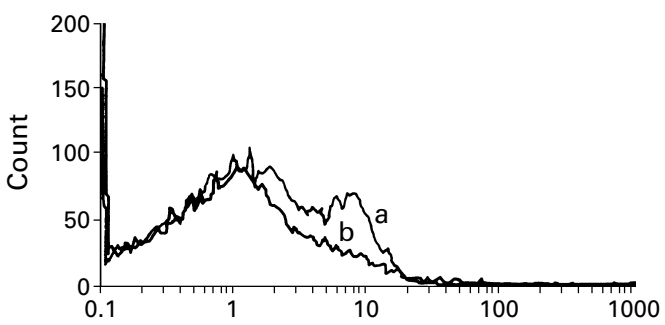

Figure 2 Immunofluorescence staining of ADAM 10 expressing COS -7 cells by a patient's serum. This histogram demonstrates that COS-7 cells transfected with the full length of $A D A M 10$ are stained positively by $F Y$ serum (a). In contrast, COS-7 cells transfected with pCDNA1.1/Amp alone were unstained with FY serum (b).

(MW 30 000) aldolase (MW 42 400) bovine albumin (MW 66 000), and phosphorylase B from rabbit muscle (MW 97 400). Proteins were electrophoretically transferred onto nitrocellulose membrane. Proteins were detected by immunoblotting, using a patient's serum, peroxidase conjugated goat antihuman IgG antibody (Sigma ImmunoChemicals, lot 094H-4810, St Louis, MO), and stained with 4CN PLUS for chromogenic detection of horseradish peroxidase (NEM Life Science Products, Boston, MA).

ISOLATION AND CHARACTERISATION OF CDNA Serum from FY (obtained in March 1995) was used for immunoscreening of the $\lambda$ TriplEX human lung cDNA library (Clonetech, Palo Alto, CA) according to the manufacturer's instructions. Two positive cDNA clones were selected from $3 \times 10^{5}$ clones, and the nucleotide sequencing was conducted using an Automated Laser Fluorescent ALF sequencer (Pharmacia LKB Biotechnology AB, Uppsala, Sweden). Both sequences appeared to be identical to the partial sequence of human ADAM 10 cDNA found by the computer homology search. Therefore, full length cDNA was isolated from original lung cDNA library using a partial cDNA as a probe. The full length ADAM 10 cDNA was subcloned to pCDNA1.1/Amp (Invitrogen, San Diego, CA) and transfected into COS-7 cells by the DEAE-dextran method. After 48 hours, the cells were incubated with FY serum or control human serum. After incubating with rabbit antihuman IgG conjugated with FITC, the cells were analysed using EPICS Profile II analyser and EPICS Elite software (Coulter, Hialeah, FL).

\section{Results}

Figure 1 shows western immunoblot analysis using a patient's serum against lysates of PC9, A549 and SAEC cell lines. Three antigens that have different molecular weights were demonstrated. There may be two bands in lane 3 of the figure 1 . However, as the upper band is very faint, this band is considered to be a nonspecific band and the band that had a molecular weight of $62 \mathrm{kDa}$ was clearly detected in the SAEC cell line. In the A549 cell line, the band that had a higher molecular weight $(54 \mathrm{kDa})$ was previously identified to be cytokeratin $8 .{ }^{11}$ 
The band that had a lower molecular weight (48 kDa) in A549 and PC9 cell lines was not characterised.

Both positive cDNA clones appeared to be a partial ADAM $10 \mathrm{cDNA}$ that encoded the 3 '-end of cDNA by BLAST search. As figure 2 shows, COS-7 cells transfected with the full length of ADAM 10 were stained positively by FY serum. In contrast, COS-7 cells transfected with pCDNA1.1/Amp alone were unstained with FY serum. Any significant staining was not observed with the control human serum.

\section{Discussion}

In this study, we demonstrated that one of the antibodies against SAEC cells in a patient with pulmonary fibrosis associated with dermatomyositis was anti-ADAM 10 antibody.

ADAM 10 is a protein first purified from bovine brain based on its ability to cleave major basic proteins. ${ }^{10-14}$ ADAM 10 , is a membrane linked protein with several domains including a metalloproteinase domain, a potential integrin binding domain, a cysteine rich sequence domain, and an EGF-like sequence domain. ${ }^{10-14}$ ADAM 10 has been implicated in a wide variety of functions including basement membrane degradation and cell-cell and cellmatrix interactions. ${ }^{10-14}$ ADAM 10 contains the consensus HEXXHXXGXXH motif, which suggests that it functions as a metalloproteinase. ${ }^{12}{ }^{13}$ A wide variety of mammalian cell lines including epithelial cells and haematopoietic cells express low levels of ADAM 10 mRNA (4.5 and $3.2 \mathrm{~kb}$ transcripts) and mature polypeptide $(62 \mathrm{kDa})$ as assessed by northern analysis and western blotting. ${ }^{12} 14$

ADAM 10 is a functional metalloproteinase with gelatinase-like activity as it can completely degrade myelin basic protein and histones. ${ }^{10}$ This protein has since been found in many tissues, ${ }^{10}$ and is homologous to the Drosophila kuz protein, suggesting a role in neurogenesis. ${ }^{12}{ }^{13}$ Importantly, it has recently been demonstrated that ADAM 10 is one of the pro-tumour necrosis factor $\alpha$ ( TNF $\alpha$ ) processing enzymes, which converts pro-TNF $\alpha$ to $\mathrm{TNF} \alpha .^{11}{ }^{12}$

The significance of anti-ADAM 10 autoantibody in the pathogenesis of pulmonary fibrosis associated with dermatomyositis should be discussed. ADAM 10 is expressed in bronchoepithelial cells. The resulting antibody-antigen interaction with immune complex formation could have a significant role in the perpetuation of the disease processes, either by direct injury of epithelial cells or via local macrophage activation as they are cleared by phagocytosis. In addition, previous studies have demonstrated that potent broad spectrum inhibitors of the matrix metalloproteinases (MMPs) can prevent $T N F \alpha$ release from monocytic cell lines. ${ }^{15}$ Therefore, it is possible to speculate that the anti-ADAM 10 antibody modulates the function of the TNF $\alpha$ converting enzyme. We are now planning to make a recombinant ADAM 10 to quantify anti-ADAM 10 antibodies in sera of patients with several collagen vascular diseases.

In conclusion, our data demonstrate that anti-SAEC cell antibody in the patient's sera with pulmonary fibrosis associated with dermatomyositis was against ADAM 10. AntiADAM 10 antibody may have played a part in the process of lung injury in pulmonary fibrosis.

1 Crystal RG, Bitterman PB, Rennard SI, Hance AJ, Keogh $\mathrm{BA}$. Interstitial lung diseases of unknown cause. Disorders characterized by chronic inflammation of the lower respiratory tract. N Engl J Med 1984;310:154-66.

2 Corrin B, Dewar A, Rodriguez-Roisin R, Turner-Warwick $\mathrm{M}$. Fine structural changes in cryptogenic fibrosing alveolitis and asbestosis. J Pathol 1985;147:107-19.

3 Dreisen RB, Schwarz MI, Theofilopoulos AN, Stanford RE. Circulating immune complexes in the idiopathic interstitial Circulating immune complexes in the idiopathic

pnlinorias.
Erlinger R, Rauh G, Behr J, Schumacher U, Welsch U, Zolliner N. Similar frequency of autoantibodies against pneumocytes type II and Clara cells in patients with interstitial lung disease and healthy persons. Klin Wochenschr 1991;69:297-302.

5 Meliconi R, Bestagno M, Sturani C, Negri C, Galavotti V, Sala C, et al. Autoantibodies to DNA topoisomerase II in cryptogenic fibrosing alveolitis and connective tissue diseases. Clin Exp Immunol 1989;76:184-9.

6 Ueda T, Ohta K, Suzuki N, Yamaguchi $M$, Hirai K, Horiuchi $\mathrm{T}$, et al. High prevalence of serum antibodies to hepatitis C virus in idiopathic pulmonary fibrosis. Am Rev Respir Dis 1992;146:266-8.

7 Wallace WAH, Roberts SN, Caldwell $\mathrm{H}$, Thornton $\mathrm{E}$ Greening AP, Lamb D, et al. Circulating antibodies to lung protein(s) in patients with cryptogenic fibrosing alveolitis. Thorax 1994;49:218-24.

8 Wallace WAH, Schofiels JA, Lamb D, Howie SEM. Localization of a pulmonary autoantigen in cryptogenic fibrosing alveolitis. Thorax 1994;49:1139-45.

9 Dobashi N, Fujita J, Ohtsuki Y, Yamadori I, Yoshinouchi T, Kamei T, et al. Detection of anti-cytokeratin 8 antibody in sera of the patients with cryptogenic fibrosing alveolitis and pulmonary fibrosis associated with collagen vascular disorpulmonary fibrosis associated

10 Chantry A, Glynn P. A novel metalloproteinase originally isolated from brain myelin membranes is present in many tissues. Biochem J 1990;268:245-8.

11 Rosendahl MS, Ko SC, Long DL, Brewer MT, Rosenzweig $\mathrm{B}$, Hedl E, et al. Identification and characterization of a pro-tumor necrosis factor- $\alpha$-processing enzyme from the ADAM family of zinc metalloproteases. J Biol Chem 1997; 272:24588-93.

12 Lunn CA, Fan X, Dalie B, Miller K, Zavodny PJ, Narula SK, et al. Purification of ADAM 10 from bovine spleen as a TNF $\alpha$ convertase. FEBS Lett 1997;400:333-5.

13 Wen C, Metzstein MM, Greenwald I. SUP-17, a Caenorhabditis elegans ADAM protein related to DroCaenorhabditis elegans ADAM protein related to Drosophila KUZBANIAN, and its role in LIN

14 Wu E, Croucher PI, McKie N. Expression of members of the novel membrane linked metalloproteinase family ADAM in cells derived from a range of haematological malignancies. Biochem Biophys Res Commun 1997;235: 437-42.

15 Mohler KM, Sleath PR, Fitzner JN, Cerretti DP, Alderson M, Kerwar SS, et al. Protection against a lethal dose of endotoxin by an inhibitor of tumour necrosis factor processing. Nature 1994;370:218-20. 